OPEN ACCESS

Edited by:

Abdur Rauf,

University of Swabi, Pakistan

Reviewed by:

Khristina Judan Cruz, Central Luzon State University, Philippines

Anees Ahmed Khalil,

University of Lahore, Pakistan

*Correspondence:

Jing Dong

dongjingletter@163.com

Xiaohui Ai

aixh@yfi.ac.cn

Specialty section:

This article was submitted to Experimental Pharmacology and

Drug Discovery,

a section of the journal

Frontiers in Pharmacology

Received: 05 August 2021 Accepted: 15 September 2021 Published: 28 September 2021

Citation:

Dong J, Zhang D, Li J, Liu Y, Zhou S, Yang Y, Xu N, Yang Q and Ai X (2021) Genistein Inhibits the Pathogenesis of Aeromonas hydrophila by Disrupting Quorum Sensing Mediated Biofilm Formation and Aerolysin Production.

Front. Pharmacol. 12:753581. doi: 10.3389/fphar.2021.753581

\section{Genistein Inhibits the Pathogenesis of Aeromonas hydrophila by Disrupting Quorum Sensing Mediated Biofilm Formation and Aerolysin Production}

\author{
Jing Dong ${ }^{1 *}$, Defu Zhang ${ }^{2}$, Jianrong $L^{2}{ }^{2}$, Yongtao $L_{i u}{ }^{1}$, Shun Zhou ${ }^{1}$, Yibin Yang ${ }^{1}$, Ning $X u^{1}$, \\ Qiuhong Yang ${ }^{1}$ and Xiaohui $\mathrm{Ai}^{1}{ }^{\text {* }}$ \\ ${ }^{1}$ Yangtze River Fisheries Research Institute, Chinese Academy of Fishery Sciences, Wuhan, China, ${ }^{2}$ College of Food Science and \\ Engineering, Bohai University, Jinzhou, China
}

Aeromonas hydrophila is an opportunistic pathogen that is responsible for a variety of infectious diseases both in human and animals, particularly aquatic animals. Moreover, the pathogen has become a foodborne pathogen by transmitting from seafood to human. The abuse of antibiotics in aquaculture results in the emergence of antibiotic resistance and treatment failure. Therefore, novel approaches are urgently needed for managing resistant A. hydrophila associated infections. Aerolysin, an essential virulence factor of pathogenic A. hydrophila strain, has been identified as target developing novel drugs against pathogenesis of $A$. hydrophila. In the present study, genistein, without anti- $A$. hydrophila activity, was identified that could decrease the production of aerolysin and biofilm formation at a dose-dependent manner. Transcription of aerolysin encoding gene aerA and quorum sensing related genes ahyl and ahy $R$ was significantly down-regulated when co-cultured with genistein. Cell viability studies demonstrated that genistein could significantly improve aerolysin mediated A549 cell injury. Furthermore, genistein could provide a remarkable protection to channel catfish infected with $A$. hydrophila. These findings indicate that targeting quorum sensing and virulence can be a useful approach developing drugs against $A$. hydrophila infections in aquaculture. Moreover, genistein can be chosen as a promising candidate in developing drugs against $A$. hydrophila.

Keywords: Aeromonas hydrophila, quorum sensing, genistein, anti-virulence, natural compound

\section{INTRODUCTION}

Aquatic products have become one of the major sources of high quality proteins for human, playing critical role in global food security, particularly in developing countries. China has become the largest producer and exporter of aquatic products all over the world since 2015 (Mo et al., 2018). However, diseases caused by bacterial pathogens threatened the healthy development of aquaculture industry and safety of aquatic products. Therefore, controlling of bacterial infection in aquaculture can not only reduce the mortality of cultured fish, but decrease the risks of foodborne infection. Aeromonas hydrophila (A. hydrophila), the leading cause of bacterial infections in freshwater aquaculture, is widely distributed in nature, particular aquatic environments (Velazquez et al., 2001). The bacterium is responsible for a number of diseases in aquatic animals, such as hemorrhagic septicemia, exophthalmia and dropsy (Patel et al., 2017). Moreover, A. hydrophila can be transmitted from 
<smiles>O=c1c(-c2ccc(O)cc2)coc2cc(O)cc(O)c12</smiles>

Genistein (Spagnuolo et al., 2015)
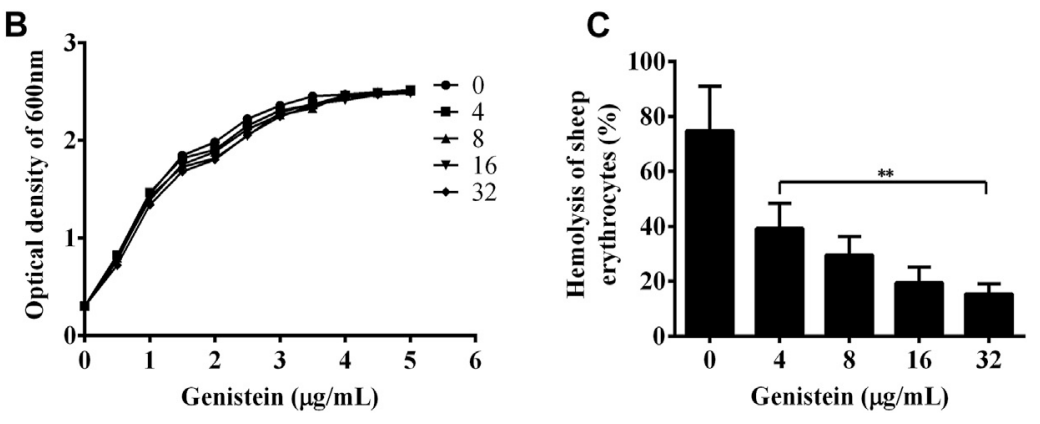

FIGURE 1 | Inhibitory effects of genistein on hemolytic activity of bacterial supernatants co-cultured with genistein. (A), chemical structure of genistein; (B), Growth curves of $A$. hydrophila XS-91-4-1 plus indicated concentrations of genistein; (C), genistein inhibits hemolytic activity of bacterial supernatants co-cultured with genistein, $1 \%$ Triton X-100 treated red blood cells represented $100 \%$ hemolytic activity, three independent hemolytic assays were performed and data in Figure $\mathbf{1 C}$ were mean value $\pm \mathrm{SD},{ }^{*} p<0.05$ and ${ }^{* *} p<0.01$ when compared with the genistein-free supernatants.

uncooked aquatic food to human, and has been considered as a foodborne bacteria associated with several human infections (Kuijper et al., 1990; Janda, 1991). Antibiotics are the main approach treating bacterial infections. However, the emergence of antibiotic resistance limits the use of antibiotics and results in treatment failure. Therefore, antibiotic alternative strategies are needed to overcome resistant $A$. hydrophila strains.

Virulence factors are involved in the infectious processes such as colonization, invasion and persistence in the hosts (Fleitas Martinez et al., 2019). Therefore, anti-virulence strategies have been identified as a novel approach in developing drugs against resistant bacterial infections. The pathogenesis of $A$. hydrophila is determined by the amount of virulence factors (Zhao et al., 2020). Among which, aerolysin has been best characterized and is considered as one of the most critical virulence factors for establishing infections (Chakraborty et al., 1987; Fivaz et al., 2001). Aerolysin is secreted as an inactive precursor naming proaerolysin, the activity of the toxin will release after cleaving the 43 residues at the $\mathrm{C}$-terminus of the protein by trypsin or furin (Iacovache et al., 2011). After activation, the toxin can form a homo-heptameric pore on target cells and results in cell death, a number of Mammalian cells are sensitive to the toxin (Wuethrich et al., 2014). Therefore, aerolysin has been identified as an ideal target developing drugs against $A$. hydrophila infections. Moreover, biofilm formation and the expression of aerolysin and several other virulence factors are regulated by quorum sensing (QS) system (Rama Devi et al., 2016). A number of studies have demonstrated that anti-QS compounds are a useful approach to overcome the bacterial resistance problem (Ding et al., 2017; Patel et al., 2017; Ding et al., 2018).

Herbal medicine and natural compounds have been used as a source of medicine and make a significant contribution to the pharmaceutical industry (Machado et al., 2020). Genistein (Figure 1A), an isoflavone compound, can be isolated from most of leguminous plant foods (Spagnuolo et al., 2015). Soybean has been reported with the highest concentrations of genistein. Previous studies have demonstrated that genistein showed a variety of biological activities, including anti-cancer, anti-inflammation, anti-apoptosis and anti-oxidation activities
(Wei et al., 1993; Ruiz-Larrea et al., 1997; Li et al., 2006). However, there is little knowledge of genistein against bacterial pathogens. In the present study, we found that genistein had no role on $A$. hydrophila growth at concentrations lower than $512 \mu \mathrm{g} / \mathrm{ml}$, could reduce the production of aerolysin and biofilm formation of $A$. hydrophila by inhibiting the QS system. Moreover, genistein could provide a significant protection to A549 cells and fish infected with A. hydrophila.

\section{MATERIALS AND METHODS}

\subsection{Microorganism and Reagents}

A. hydrophila strain XS-91-4-1 was kindly provided by Prof. Aihua $\mathrm{Li}$ at Institute of Hydrobiology, Chinese Academy of Sciences. Genistein (CAS No. 446-72-0) with purity of $99.5 \%$ was purchased from National Institutes for Food and Drug Control (Beijing, China). Enrofloxacin (CAS No. 93106-60-6) was purchased from Sigma-Aldrich (St. Louis, MO, United States). For in vitro studies, Genistein and enrofloxacin were prepared as stock solutions at concentrations of $40.96 \mathrm{mg} /$ $\mathrm{ml}$ by DMSO. For in vivo study, genistein was dissolved in sterile PBS.

\subsection{Determination of Minimal Inhibitory Concentrations of Enrofloxacin and Genistein}

Micro-dilution method was used to assess the MICs of enrofloxacin and genistein against $A$. hydrophila XS-91-4-1 under the guidance of CLSI (2009). In brief, enrofloxacin and genistein were diluted at 2-folder by MHB medium in 96-well plate to reach concentrations ranging from 32 to $0.125 \mu \mathrm{g} / \mathrm{ml}$ and 512 to $2 \mu \mathrm{g} / \mathrm{ml}$, respectively. Bacterial cells were collected by centrifugation and re-suspended in MHB medium to obtain a density of about $5 \times 10^{5} \mathrm{CFU} / \mathrm{ml}$ by McFarland standards. Then $100 \mu \mathrm{l}$ of bacterial suspension was added to each well and incubated for $8-20 \mathrm{~h}$ at $28^{\circ} \mathrm{C}$. To evaluate the impact of DMSO on bacterial growth, DMSO was added to the positive 
control group. The MIC value of each drug was defined as the lowest concentrations with no visible bacterial growth.

\subsection{Growth Curves}

Growth curves assay was carried out to determine the impact of genistein on bacterial growth. A. hydrophila XS-91-4-1was culture in Brain-heart infusion (BHI) medium overnight, then the bacterial culture was sub-inoculated to $100 \mathrm{ml}$ fresh $\mathrm{BHI}$ medium at a ratio of 1:100. The culture was aliquoted into 5 flasks at a volume of $20 \mathrm{ml}$ when the optical density at $600 \mathrm{~nm}$ $\left(\mathrm{OD}_{600 \mathrm{~nm}}\right)$ of the culture reached to 0.3 . Then different concentrations of genistein were added to each flask and incubated for further $5 \mathrm{~h}$ at $28^{\circ} \mathrm{C}$. A spectrophotometer was used to measure the $\mathrm{OD}_{600 \mathrm{~nm}}$ values of each flask every $30 \mathrm{~min}$.

\subsection{Hemolytic Activity Assay}

Hemolytic activity assay was performed as described elsewhere (Dong et al., 2017). Briefly, bacterial cultures supplemented with indicated concentrations of genistein at $28^{\circ} \mathrm{C}$ in a shaker incubator to obtain the $\mathrm{OD}_{600 \mathrm{~nm}}$ to 1.5 . Then bacterial supernatants were acquired by centrifugation. The hemolytic reaction system was brought up by $875 \mu$ l hemolytic buffer, $100 \mu \mathrm{l}$ trypsin-activated supernatant and $25 \mu \mathrm{l}$ sheep red blood cells $\left(5 \times 10^{6} \mathrm{cells} / \mathrm{ml}\right) .1 \%$ Triton X-100 treated group served as positive control group. The mixtures were centrifuged to remove the unlyzed red blood cells after an incubation for $20 \mathrm{~min}$ at $37^{\circ} \mathrm{C}$. Then the hemolytic activities of different treatment were evaluated by measuring the absorption at $\mathrm{OD}_{543 \mathrm{~nm}}$ of the supernatants.

\subsection{Western-Blotting Assay}

Supernatants obtained from hemolytic activity assay were used for Western-blotting assay. Supernatants were boiled for $10 \mathrm{~min}$ after mixed with laemmli sample buffer. Samples were centrifuged and loaded to a sodium dodecyl sulfate (SDS)polyacrylamide (12\%) gel. Then proteins in the gel were transferred to a polyvinylidene fluoride membrane by a semidry transfer cell. The membrane was incubated with a primary anti-aerolysin polyclonal antibody after blocked with $5 \%$ non-fat milk at room temperature. Following incubated with HRPconjugated secondary goat anti-rabbit antiserum, proteins the membrane was detected by a Supper ECL Western Blotting Substrate.

\subsection{Biofilm Formation Assay}

Biofilm formation assay was performed in 96 well plates according to previous study with some modification (Tanhay Mangoudehi et al., 2020). An overnight bacterial culture was subinoculated to fresh BHI medium to obtain a $\mathrm{OD}_{600 \mathrm{~nm}}$ of 1.0. The bacterial culture was diluted with fresh medium at a ratio of 1:20 and added to each well with various concentrations of genistein. The mixtures were further incubated without shaking at $37^{\circ} \mathrm{C}$ for $24 \mathrm{~h}$. Wells filled with fresh medium was served as negative control, while wells filled with bacterial suspension with an indicated concentration of DMSO was served as positive control. Before quantification, the values of $\mathrm{OD}_{600 \mathrm{~nm}}$ were determined to confirm all the wells were at stationary phase. The medium and unattached bacteria were removed and then washed with PBS to remove any remaining planktonic bacterial cells. The biofilms in each well were stained with $0.5 \%$ crystal violet for $30 \mathrm{~min}$ after air-dried. After washing, the dye was released by addition of $30 \%$ glacial acetic acid. The quantification of the biofilm was determined by measuring the absorption at $570 \mathrm{~nm}$ by a microplate reader.

\subsection{Microscopic Assay of Biofilm Formation}

For microscopic assays, biofilms were grown on glass slides in a 24 well cell plate with indicated concentrations of genistein at $37^{\circ} \mathrm{C}$ for $24 \mathrm{~h}$. The unattached bacterial cells were removed by washing with distilled water for three times and then treated as described below. For light microscopic analysis, the glass slides were air-dried and stained with $0.5 \%$ crystal violet for $3 \mathrm{~min}$. After washing and drying, the biofilms on the glass slides were directly detected by a light microscope at a magnification of $\times 400$ and captured by an inbuilt digital camera.

\subsection{Quantitative Real-Time PCR}

Bacterial cells co-cultured with indicated concentrations of genistein were acquired when $\mathrm{OD}_{600 \mathrm{~nm}}$ of the cultures reached to 1.5. Then total RNA of the bacteria was extracted by a MolPure Bacterial RNA Kit. After removing the excessed DNA, total RNA was used to synthesize cDNA by a PrimeScript RT Master Mix. Then qRT-PCR was performed on a CFX96 Touch Real-Time PCR Detection System (Bio-Rad, California, United States). Primer pairs of detecting genes were listed in Table 1. The relative expression levels of each gene was analyzed by $2^{-\Delta \Delta \mathrm{Ct}}$ method.

\subsection{Cell Viability Assays}

The protective effect of genistein on aerolysin induced cell injury was evaluated by A549 cells according to previous study. Briefly, A549 cells obtained from ATCC was cultured in DMEM medium supplemented with $10 \%$ fetal bovine serum at $37^{\circ} \mathrm{C}$ with $5 \% \mathrm{CO}_{2}$. Cells at a density of $1.5 \times 10^{5}$ per well were seeded into a 96-well cell plate and were further incubated overnight. Then bacterial supernatants used in hemolytic assay were added to each well and co-cultured with A549 cells for further $2 \mathrm{~h}$. After incubation, cell supernatants were obtained and were applied for LDH release assay using a LDH Cytotoxicity Assay Kit. Cells were washed by sterile PBS for three times and were stained by LIVE/DEAD regents. Live cells were stained with green, while dead cells were red, images were captured by a fluorescence microscope.

\subsection{Ethics Statement}

Animal studies were performed at fishery drugs clinical trials center of Yangtze River Fisheries Research Institute under the guidance of the experimental practices and standards developed by the Animal Welfare and Research Ethics Committee. The experimental protocols were approved and supervised by the animal care committee (Permit No. YFI-2020DJ-019).

\subsection{Experimental Therapeutics}

60 healthy channel catfish was divided into 3 groups, and was maintained in $100 \mathrm{~L}$ glass tanks for 7 days before bacterial challenge. XS-91-4-1 was cultured in BHI medium at $28^{\circ} \mathrm{C}$ to the mid-log phase, bacterial cells were obtained by centrifugation. 
TABLE 1 | Primer pairs used in qPCR assay.

\begin{tabular}{|c|c|c|c|}
\hline Primer & Sequence $\left(5^{\prime}-3^{\prime}\right)$ & PCR amplicon (bp) & References \\
\hline aerA-F & TCTACСАССАССТСССТGTC & 218 & Dong et al. (2017) \\
\hline aerA-R & GACGAAGGTGTGGTTCCAGT & & \\
\hline ahyR-F & TITACGGGTGACCTGATTGAG & 206 & Patel et al. (2017) \\
\hline ahyR-R & CCTGGATGTCCAACTACATCTT & & \\
\hline ahyl-F & GTCAGCTCCCACACGTCGTT & 202 & Dong et al. (2020) \\
\hline ahyl-R & GGGATGTGGAATCCCACCGT & & \\
\hline $16 S$ rRNA-F & TAATACCGCATACGCCCTAC & 164 & Dong et al. (2017) \\
\hline $16 \mathrm{~S}$ rRNA-R & ACCGTGTCTCAGTTCCAGTG & & \\
\hline
\end{tabular}

After washed by sterile PBS for 3 times, the concentration of bacterial cells was adjusted to $1.5 \times 10^{8} \mathrm{cfu} / \mathrm{ml}$ by a McFarland standard. Fish in positive group and genistein treated group were intraperitoneally injected with $200 \mu$ l bacterial suspension, while fish in negative group were injected with $200 \mu \mathrm{l}$ sterile PBS. Then fish in genistein treated group were administered with $20 \mathrm{mg} / \mathrm{ml}$ genistein by a gavage needle, while fish in positive group and negative group were administered with PBS $6 \mathrm{~h}$ post infection and then 12- $\mathrm{h}$ intervals for 3 days. Deaths in each group were observed every $24 \mathrm{~h}$ for 8 days.

\subsection{Statistical Analysis}

Data in hemolysis, biofilm formation, relative gene expression and LDH assay were analyzed by Student's $t$-test. The survival rate was analyzed by Kaplan-Meier estimates and log-rank test. $p<0.05$ indicates statistical significance.

\section{RESULTS}

\subsection{Effect of Genistein on Bacterial Growth} The MIC of genistein against A. hydrophila XS-91-41 was higher than $512 \mu \mathrm{g} / \mathrm{ml}$, while $4 \mu \mathrm{g} / \mathrm{ml}$ for enrofloxacin when determined by micro-dilution method. The results indicated that genistein had no inhibitory effect against $A$. hydrophila under our experimental conditions. Moreover, the results of growth curves assay (Figure 1B) showed that genistein had no influence to bacterial growth at concentrations ranging from 32 to $4 \mu \mathrm{g} / \mathrm{ml}$ in $5 \mathrm{~h}$.

\subsection{Effect of Genistein on Hemolysis of Bacterial Supernatants}

The MIC and growth curves assays had demonstrated that there was no role of genistein against bacterial growth under our experimental concentrations. However, the hemolytic activity assay showed that genistein could inhibit the hemolysis induced by bacterial supernatants at a dose dependent manner following the addition of genistein in bacterial cultures. As shown in Figure 1C, the hemolysis decreased to $39.23 \pm 7.46,29.52 \pm 5.55,19.47 \pm 4.60$, and $15.42 \pm 3.03 \%$ when co-cultured with genistein at concentrations of $4,8,16,32 \mu \mathrm{g} / \mathrm{ml}$, while the hemolysis of drug-free group was $74.73 \pm 13.29 \%$. The hemolytic activity of the supernatant was significantly reduced by addition of genistein at concentrations of $4 \mu \mathrm{g} / \mathrm{ml}$ and above. To analyze the inhibitory effect of genistein on the production of aerolysin in the supernatants, Western blot assay was performed. As desired, the levels of aerolysin in the supernatant were sequentially decreased following the increasing concentrations of genistein (Figure 2). As shown in Figure 2, there was only a little aerolysin was detected when genistein reached to $32 \mu \mathrm{g} / \mathrm{ml}$. Taken together, the results revealed that genistein could reduce the hemolytic activity of bacterial supernatants when co-cultured with A. hydrophila at no-inhibitory concentrations by decreasing the production of aerolysin.

\subsection{Inhibitory Effect of Genistein on Biofilm Architecture}

The inhibitory effect of genistein on biofilm formation of $A$. hydrophila was determined. As shown in Figure 3A, the addition of genistein could reduce the quantity of biofilm at a dose dependent manner when determined by crystal violet staining. Biofilm formation of A. hydrophila was significantly inhibited when cocultured with genistein at concentrations of $8 \mu \mathrm{g} / \mathrm{ml}$ and above (Figure 3A). The amount of biofilm decreased to $31.86 \%$ in group co-cultured with $32 \mu \mathrm{g} / \mathrm{ml}$ genistein compared with drug free group. Moreover, the inhibitory effect of genistein on biofilm formation was analyzed microscopically by testing bacterial biofilms on glass slides with indicated concentrations of genistein. As shown in Figures 3B-F, a dense biofilm was observed on the control glass slide, while a visible reduction of biofilm in the glass slide with $4 \mu \mathrm{g} / \mathrm{ml}$ genistein and above (Figure 3F).

\subsection{Effect of Genistein on Transcription of Related Genes}

Aerolysin expression and biofilm formation of $A$. hydrophila were regulated by QS system. The results of hemolytic activity assay and biofilm formation assay indicated that genistein might be a QS inhibitor. Therefore, the transcription levels of aerolysin encoding gene aerA and QS regulators ahyI and $a h y R$ were analyzed by qPCR. As shown in Figure 4, the aerA gene was 8.39-fold down-regulated plus $32 \mu \mathrm{g} / \mathrm{ml}$ genistein compared with drug-free group, while 7.61 -fold and 10.55 -fold for ahyI and $a h y R$, respectively (Figure 4).

\subsection{Effect of Genistein on Aerolysin Mediated Cell Injury}

A549 cells was used to evaluate the protective effect of genistein on aerolysin induced cell injury. As shown in Figure 5A, cells without any treatment were stained green, indicating live cells. 


\section{$-\cdots-$ \\ $\begin{array}{llllll}0 & 4 & 8 & 16 & 32 & 0\end{array}$ \\ Genistein $(\mu \mathrm{g} / \mathrm{mL})$}

FIGURE 2 | Detection of aerolysin production in bacterial supernatant with genistein by Western-blot.

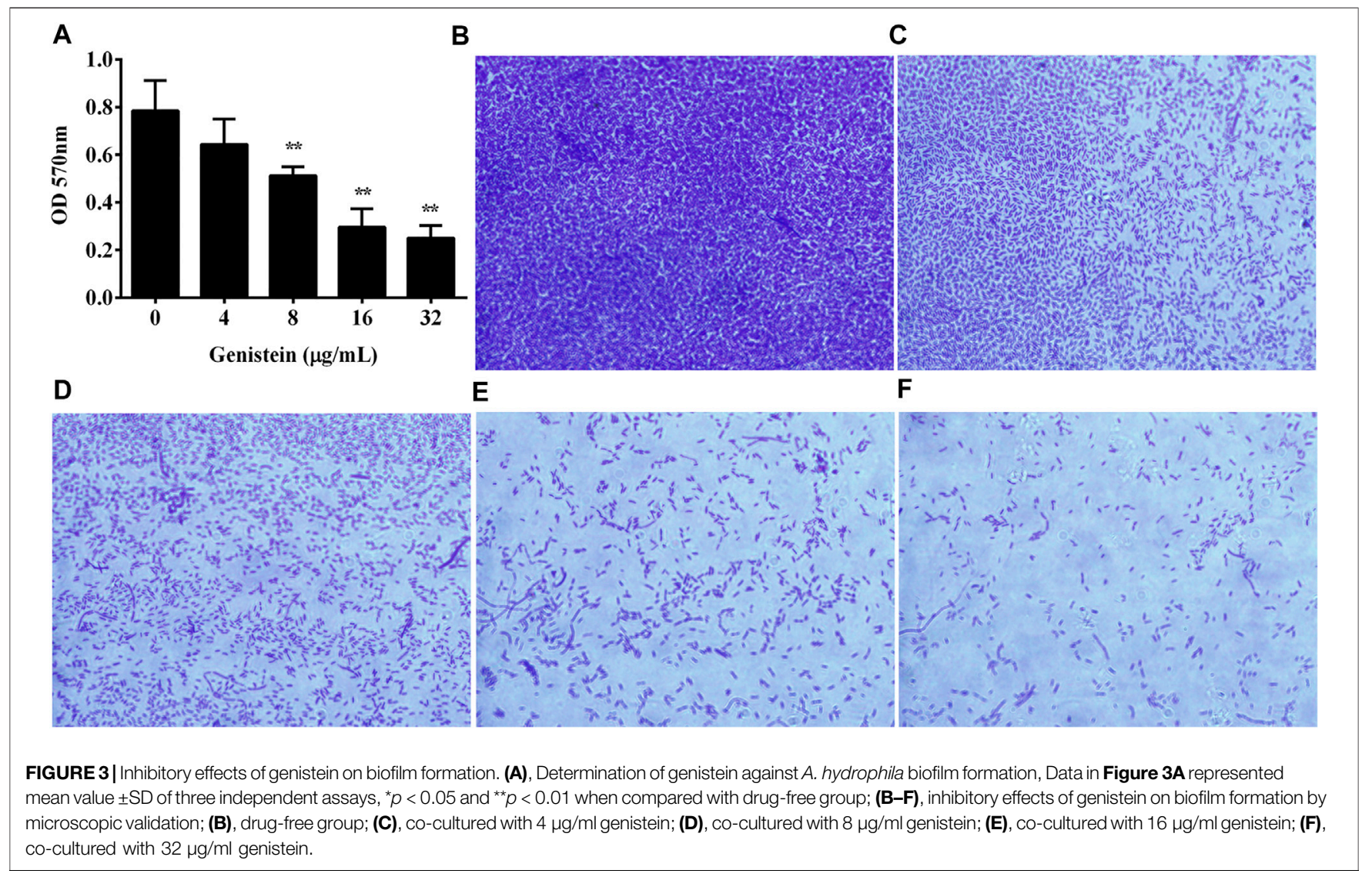

Dead cells staining red were observed when treated with drug-free bacterial supernatant (Figure 5B). The quantity of dead cells in genistein treated group showed a visible decrease compared with drug-free group (Figure 5C). The results indicated that genistein could reduce cell death caused by aerolysin. Moreover, cell viability was determined by $\mathrm{LDH}$ release assay. As shown in Figure 5D, genistein could reduce $\mathrm{LDH}$ release at a dose dependent manner. LDH release of A549 cells decreased to $25.94 \pm 3.92 \%$ in group co-cultured with bacterial supernatant plus $32 \mu \mathrm{g} / \mathrm{ml}$ genistein, while $85.42 \pm 7.65 \%$ of drug-free group.

\subsection{Protective Effect of Genistein on Fish Challenged With $A$. hydrophila}

In vitro studies have demonstrated that genistein could protect A549 cells from cell death by inhibiting the expression of aerolysin and QS system. Therefore, an A. hydrophila infection model of channel catfish was established to study the in vivo protective effect of genistein. Fish challenged with $A$. hydrophila showed heamorrhage and swelling in gill and fin. Ascites in abdominal cavity and hyperaemia in liver and kidney were found by anatomy. Moreover, body weight loss was observed in positive control group by monitoring body weight every $24 \mathrm{~h}$. Deaths were occurred after $24 \mathrm{~h}$ post challenge. As shown in Figure 6, all fish in positive control group were dead at 6 days post infection, while fish in genistein treated group showed $75 \%$ survive which was statistically significant $(p<0.01)$ compared with positive control. No death was observed in negative control group during all the experimental period. The results demonstrated that treatment with genistein could significant decrease the pathogenesis of $A$. hydrophila in a channel catfish infection model. 


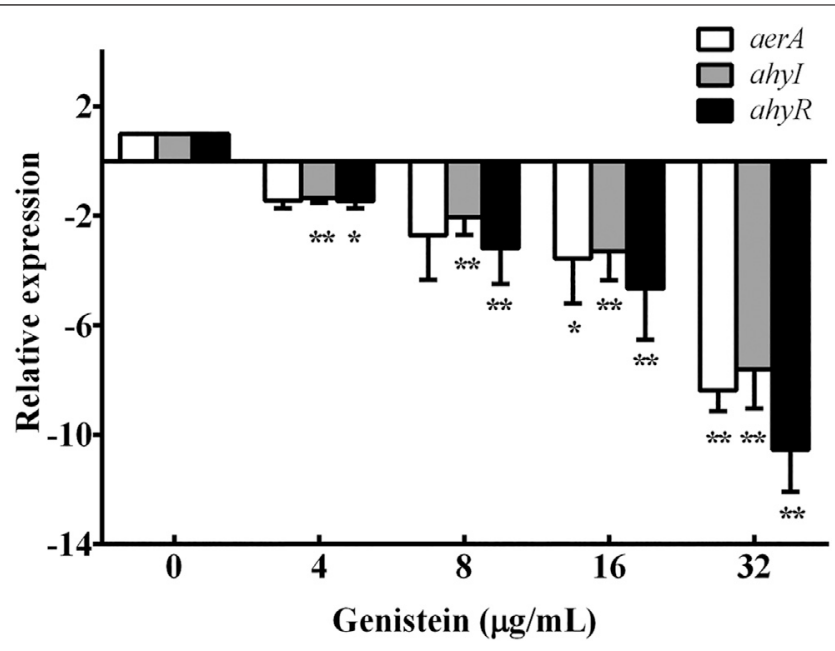

FIGURE 4 | Treatment with genistein decreased the transcription levels of aerA, ahyl and ahyR. Transcription levels of different genes were determined by qPCR assays in triplicate, data were mean value \pm SD. ${ }^{*} p<0.05$ and ${ }^{* *} p<0.01$.
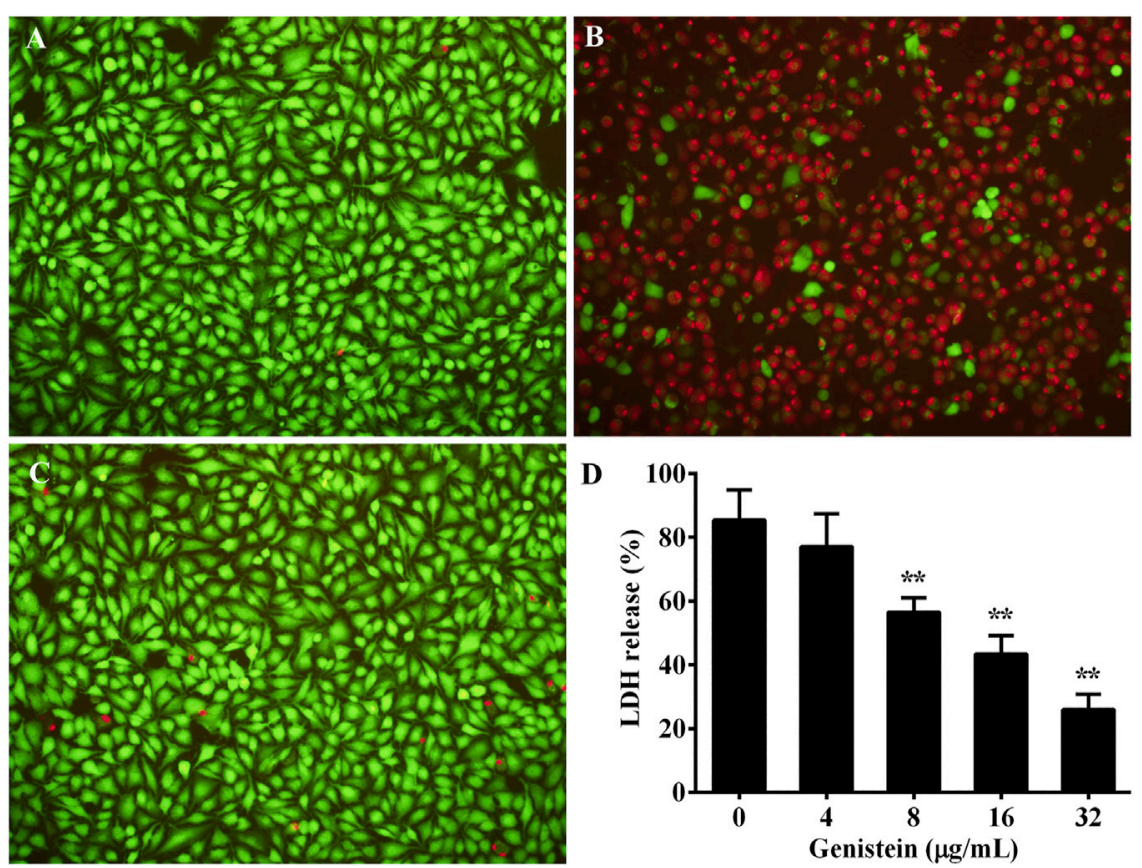

FIGURE 5|Protective effects of genistein on aerolysin mediated A549 cell injury. Cell injury induced by aerolysin was evaluated by live/dead staining assay and LDH release assay. For live/dead staining assay, live cells were stained with green, while dead were red. (A), cells without any treatment; (B), cells treated with drug-free supernatant; (C), cells treated with supernatant obtained from bacterial culture plus $32 \mu \mathrm{g} / \mathrm{ml}$ genistein; (D), LDH release of A549 cells treated with bacterial supernatants plus indicated concentrations of genistein, $\mathrm{LDH}$ assay was performed in triplicate, data were mean value \pm SD. ${ }^{*} p<0.05$ and ${ }^{* *} p<0.01$.

\section{DISCUSSION}

The growth of human population and development of agriculture has resulted in the emergence of infectious diseases (McMichael, 2004). The introduction of penicillin and other antibiotics since 1940s has significantly increased the survival rate of human suffered from bacterial infections (Coates et al., 2002).
However, antibiotic resistance observed in a few years after the deployment of antibiotics, which had become one of the biggest challenges of the 21th century (Palumbi, 2001; Rasko and Sperandio, 2010). Antibiotics were widely used in aquaculture in attempts to dealing with bacterial infections. The wide and frequent use of antibiotics in fish farming has led to the development of antibiotic resistance in aquatic bacterial 


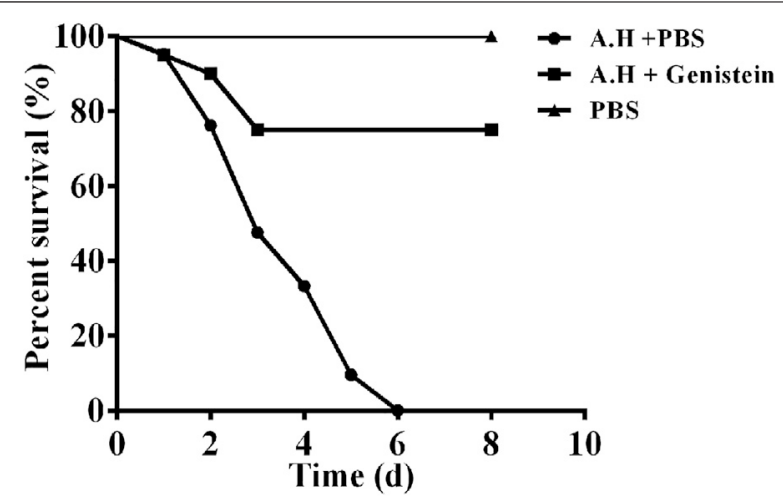

FIGURE 6 | Genistein treatment improved the survival rate of channel catfish infected with $A$. hydrophila. Fish infected with $A$. hydrophila were treated with $20 \mathrm{mg} / \mathrm{kg}$ genistein or sterile PBS every $12 \mathrm{~h}$ for 3 days, deaths were observed for 8 days after infection. Treatment with genistein could provide a significant protection to fish challenged with $A$. hydrophila when analyzed by log-rank test $(p<0.0001)$.

pathogens (Defoirdt et al., 2011). In some cases, cure failure was observed when treated bacterial infections by antibiotics. A. hydrophila, one of the most common bacterial pathogens in aquaculture environments, has been reported to be resistant to a number of antibiotics such as ciprofloxacin, tetracycline and rifampin (Hossain et al., 2018). Resistant genes of $A$. hydrophila was located on transferable plasmids and integrons which could transmit to animal and human bacterial pathogens (Defoirdt et al., 2011). Antibiotic resistant bacteria have become an important public health threat. Therefore, alternative strategies were needed to overcome microbial infections. In recent year, targeting virulence has become a novel approach for antimicrobial therapy with the increasing knowledge of bacterial pathogenesis (Clatworthy et al., 2007). Virulence factors of pathogenic bacteria are required to cause host damage and diseases. Thus, anti-virulence strategy was to decrease the disruption caused by virulence factors to the host. Then the attenuated bacterial strain will be cleared by the host immune response (Clatworthy et al., 2007). Toxins, adhesions, secretion systems and regulations of virulence can be selected as targets identifying alternative drugs combating bacterial infections. The pathogenesis of A. hydrophila was composed by toxins, structural components, secretion systems, biofilm and proteins associated with metals (Fernandez-Bravo and Figueras, 2020). Aerolysin can form a channel pore on target cells by binding to the glycophospha-tidylinositol (GPI) anchored proteins and results in cell death (Singh et al., 2008). Biofilms can increase the survival rate and contamination of bacterial pathogens in aquatic environment (Packiavathy et al., 2013), which is critical for bacterial pathogens in establishing infections and developing diseases (Tanhay Mangoudehi et al., 2020). Thus, aerolysin, the chief virulence factor, and QS, virulence regulating system, were selected as targets in drug discovery.

Herbal medicines have a long history in the treatments of human and animal diseases. Moreover, herbal medicines are the only resources for treating infectious diseases in some developing countries (Savoia, 2012). Natural compounds extracted from herbal medicines have been proven to play critical roles in the anti-infective area (Newman and Cragg, 2012). Therefore, herbal medicines have attracted the attention of researchers in developing novel drugs against bacterial infections. Bacterial infections in fish farming are one of the major concerns limiting the healthy development of aquaculture. Although the introduction of antibiotics to aquaculture decreases mortality and avoid huge economic losses in aquaculture, the therapy is limited because of the emergence of antibiotic resistance, adverse effects to aquatic environments and human health (Valladao et al., 2015). Therefore, herbal medicines with anti-bacterial, antiinflammatory, anti-oxidative and anti-parasitic activities have been widely used in aquaculture as an alternative for treating fish diseases (Valladao et al., 2015; Perez-Sanchez et al., 2018).

In the present study, natural compounds were used to screen drugs inhibiting the pathogenesis of $A$. hydrophila. As expected, genistein was found to provide a significant protection to channel catfish challenged with A. hydrophila. Genistein is well-known as a tyrosine-specific-protein kinase that can prevent a number of microorganisms into target cells (Vela et al., 2008; Siriviriyakul et al., 2020). In aquaculture, genistein has been recognized as an additive to improve body function. Fickler et al. (2019) evaluated the ability of genistein on biosynthesis of omega-3 fatty acids eicosapentaenoic acid and docosahexaenoic acid in Oncorhynchus mykiss, the results showed that the addition of genistein could stimulate the biosynthesis of docosahexaenoic acid at a moderate extent (Fickler et al., 2019). Moreover, Jourdehi et al. (2014) demonstrated that genistein could be used as an additive to diets for inducing ovary development of Huso (Jourdehi et al., 2014). Tan et al. (1998) demonstrated that genistein could be a tyrosine kinase inhibitor that postpone the internalization of A. hydrophila into host cells (Tan et al., 1998). These reports indicated that genistein could be a useful agent in aquaculture. However, there was little study of genistein against aquatic bacteria. Verdrengh et al. (2004) found that genistein could inhibit the growth of Staphylococcus aureus (S. aureus) including methicillin-resistant strains of $S$. aureus in vitro by disturbing the stabilization of the covalent topoisomerase II-DNA cleavage complex, indicating that genistein could be a potent antistaphylococcal agent (Verdrengh et al., 2004). Hong et al. (2006) 
reported that genistein had antibacterial activity to opportunistic bacterial pathogens, such as Bacillus anthracis and S. aureus at $100 \mu \mathrm{M}$ (Hong et al., 2006). Here we found that the MIC of genistein against $A$. hydrophila XS-91-4-1 was higher than $512 \mu \mathrm{g} / \mathrm{ml}$. Taken the results of growth curves together, these indicated that genistein could hardly inhibit the growth of $A$. hydrophila under our experimental conditions.

Although the antibacterial activity of genistein to different kinds of bacterial organisms was different, it has been reported as virulence inhibitors against bacterial pathogens (Oh et al., 2010; Kim and Lee, 2020; Gautam et al., 2021). Kim and Lee (2020) found that genistein-induced nitric oxide could cause apoptosislike death in Escherichia coli (Kim and Lee, 2020). Gautam et al. (2021) investigated the inhibitory effect of genistein against Acinetobacter baumannii (A. baumannii) by disrupting virulence (Gautam et al., 2021). Inhibition of biofilm, surface motility and increasing expression of polyP degrading enzyme were observed, indicating that genistein could be a potent anti- $A$. baumannii agent by inhibiting virulence. In the present study, genistein could affect the production of aerolysin in the bacterial supernatants and biofilm formation at a dose-dependent manner. Mutagenesis of $a h y I$ and $a h y R$ genes could reduce the production of exoprotease by abolishing the ability of producing QS signal N-butanoyl-L-homoserine lactone in A. hydrophila (Swift et al., 1999). QS system of pathogenic A. hydrophila involved in the regulation of biofilm formation, hemolytic activity and motility and other phenotypes (Rama Devi et al., 2016). Therefore, the transcription of aerolysin encoding gene aerA and QS related gene ahyI and ahyR were detected according to previous study (Patel et al., 2017). All three detected genes were down-regulated after treatment of genistein. The findings in qPCR assay indicated that genistein could inhibit the production of aerolysin and biofilm formation of $A$. hydrophila by inhibiting QS system. Moreover, previous studies demonstrated that drugs inhibiting aerolysin production and biofilm via inhibiting QS could decrease the pathogenesis of $A$. hydrophila in animal models (Rama Devi et al., 2016; Patel et al., 2017; Dong et al., 2020). Thus, it is reasonable to believe that genistein can protect fish infected with A. hydrophila. As desired, a significantly protective effect was achieved by administering with $20 \mathrm{mg} / \mathrm{kg}$ genistein to fish infected with $A$. hydrophila. Genistein could inhibit the pathogenesis of $A$. hydrophila XS-91-4-1 strain both in vitro and in vivo under our experimental conditions, but the expression levels of aerolysin in different sources of A. hydrophila was distinctive. Therefore, the dosage of genistein should be adjusted according to the pathogenesis of A. hydrophila if used for treatment of $A$. hydrophila associated infections. Taken together, genistein can be developed as a novel fishery drug by

\section{REFERENCES}

Chakraborty, T., Huhle, B., Hof, H., Bergbauer, H., and Goebel, W. (1987). Marker Exchange Mutagenesis of the Aerolysin Determinant in Aeromonas Hydrophila Demonstrates the Role of Aerolysin in A. HydrophilaAssociated Systemic Infections. Infect. Immun. 55 (9), 2274-2280. doi:10.1128/iai.55.9.2274-2280.1987 inhibiting the pathogenesis of $A$. hydrophila rather than affecting the growth of the bacterium. Genistein can be an alternative approach against $A$. hydrophila infections which might decrease the use of antibiotics and delay the emergence of antibiotic resistance in aquaculture. Moreover, the findings provide a novel approach identifying drugs against bacterial infections in aquaculture.

\section{CONCLUSION}

The present study investigated the pharmacological activity of genistein against the pathogenesis of $A$. hydrophila by inhibiting QS system. The results found that genistein could decrease the production of aerolysin and biofilm at concentrations without anti- $A$. hydrophila activity by inhibiting QS mechanism. Moreover, genistein could provide a protection both in vitro and in vivo against $A$. hydrophila. These findings indicated that genistein can be a promising candidate in the treatment of $A$. hydrophila infections.

\section{DATA AVAILABILITY STATEMENT}

The raw data supporting the conclusion of this article will be made available by the authors, without undue reservation.

\section{ETHICS STATEMENT}

The animal study was reviewed and approved by the Animal Welfare and Research Ethics Committee of Yangtze River Fisheries Research Institute.

\section{AUTHOR CONTRIBUTIONS}

Conceptualization, writing-review and editing, JD, JL, and XA; investigation, methodology, JD, DZ, and YL; formal analysis, QY and YY; resources, SZ; data curation, JD and NX; supervision, JL and XA; project administration, funding acquisition, JD and JL.

\section{FUNDING}

This work is supported by National Key R\&D Program of China (2019YFD0901702) and the National Nature Science Foundation of China (No. 31702368). Paradigm for Antimicrobial Therapy. Nat. Chem. Biol. 3 (9), 541-548. doi:10.1038/nchembio.2007.24

CLSI (2009). Methods for Dilution Antimicrobial Susceptibility Tests for Bacteria that Grow Aerobically-Eighth Edition: Approved Standard M07-A8. Wayne, PA, USA: CLSI.

Coates, A., Hu, Y., Bax, R., and Page, C. (2002). The Future Challenges Facing the Development of New Antimicrobial Drugs. Nat. Rev. Drug Discov. 1 (11), 895-910. doi:10.1038/nrd940 
Defoirdt, T., Sorgeloos, P., and Bossier, P. (2011). Alternatives to Antibiotics for the Control of Bacterial Disease in Aquaculture. Curr. Opin. Microbiol. 14 (3), 251-258. doi:10.1016/j.mib.2011.03.004

Ding, T., Li, T., and Li, J. (2018). Impact of Curcumin Liposomes with AntiQuorum Sensing Properties against Foodborne Pathogens Aeromonas Hydrophila and Serratia Grimesii. Microb. Pathog. 122, 137-143. doi:10.1016/j.micpath.2018.06.009

Ding, T., Li, T., Wang, Z., and Li, J. (2017). Curcumin Liposomes Interfere with Quorum Sensing System of Aeromonas Sobria and In Silico Analysis. Sci. Rep. 7 (1), 8612. doi:10.1038/s41598-017-08986-9

Dong, J., Ding, H., Liu, Y., Yang, Q., Xu, N., Yang, Y., et al. (2017). Magnolol Protects Channel Catfish from Aeromonas Hydrophila Infection via Inhibiting the Expression of Aerolysin. Vet. Microbiol. 211, 119-123. doi:10.1016/ j.vetmic.2017.10.005

Dong, J., Zhang, L., Liu, Y., Xu, N., Zhou, S., Yang, Q., et al. (2020). Thymol Protects Channel Catfish from Aeromonas Hydrophila Infection by Inhibiting Aerolysin Expression and Biofilm Formation. Microorganisms 8 (5), 636. doi:10.3390/microorganisms8050636

Fernández-Bravo, A., and Figueras, M. J. (2020). An Update on the Genus Aeromonas: Taxonomy, Epidemiology, and Pathogenicity. Microorganisms 8 (1), 129. doi:10.3390/microorganisms8010129

Fickler, A., Staats, S., Rimbach, G., and Schulz, C. (2019). Screening Dietary Biochanin A, Daidzein, Equol and Genistein for Their Potential to Increase DHA Biosynthesis in Rainbow trout (Oncorhynchus mykiss). Plos One 14 (1), e0210197. doi:10.1371/journal.pone.0210197

Fivaz, M., Abrami, L., Tsitrin, Y., and van der Goot, F. G. (2001). Aerolysin from Aeromonas Hydrophila and Related Toxins. Curr. Top. Microbiol. Immunol. 257, 35-52. doi:10.1007/978-3-642-56508-3_3

Fleitas Martínez, O., Cardoso, M. H., Ribeiro, S. M., and Franco, O. L. (2019). Recent Advances in Anti-virulence Therapeutic Strategies with a Focus on Dismantling Bacterial Membrane Microdomains, Toxin Neutralization, Quorum-Sensing Interference and Biofilm Inhibition. Front. Cel Infect. Microbiol. 9, 74. doi:10.3389/fcimb.2019.00074

Gautam, L. K., Sharma, P., and Capalash, N. (2021). Attenuation of Acinetobacter Baumannii Virulence by Inhibition of Polyphosphate Kinase 1 with Repurposed Drugs. Microbiol. Res. 242, 126627. doi:10.1016/ j.micres.2020.126627

Hong, H., Landauer, M. R., Foriska, M. A., and Ledney, G. D. (2006). Antibacterial Activity of the Soy Isoflavone Genistein. J. Basic Microbiol. 46 (4), 329-335. doi:10.1002/jobm.200510073

Hossain, S., De Silva, B. C. J., Dahanayake, P. S., and Heo, G. J. (2018). Characterization of Virulence Properties and Multi-Drug Resistance Profiles in Motile Aeromonas Spp. Isolated from Zebrafish (Danio rerio). Lett. Appl. Microbiol. 67 (6), 598-605. doi:10.1111/lam.13075

Iacovache, I., Degiacomi, M. T., Pernot, L., Ho, S., Schiltz, M., Dal Peraro, M., et al. (2011). Dual Chaperone Role of the C-Terminal Propeptide in Folding and Oligomerization of the Pore-Forming Toxin Aerolysin. Plos Pathog. 7 (7), e1002135. doi:10.1371/journal.ppat.1002135

Janda, J. M. (1991). Recent Advances in the Study of the Taxonomy, Pathogenicity, and Infectious Syndromes Associated with the Genus Aeromonas. Clin. Microbiol. Rev. 4 (4), 397-410. doi:10.1128/CMR.4.4.397

Kim, H., and Lee, D. G. (2020). Nitric Oxide-Inducing Genistein Elicits Apoptosis-like Death via an Intense SOS Response in Escherichia coli. Appl. Microbiol. Biotechnol. 104 (24), 10711-10724. doi:10.1007/s00253020-11003-1

Kuijper, E. J., van Alphen, L., Peeters, M. F., and Brenner, D. J. (1990). Human Serum Antibody Response to the Presence of Aeromonas Spp. In the Intestinal Tract. J. Clin. Microbiol. 28 (3), 584-590. doi:10.1128/jcm.28.3.584-590.1990

Li, D. Y., Tao, L., Liu, H., Christopher, T. A., Lopez, B. L., and Ma, X. L. (2006). Role of ERK1/2 in the Anti-apoptotic and Cardioprotective Effects of Nitric Oxide after Myocardial Ischemia and Reperfusion. Apoptosis 11 (6), 923-930. doi:10.1007/s10495-006-6305-6

Machado, I., Silva, L. R., Giaouris, E. D., Melo, L. F., and Simões, M. (2020). Quorum sensing in Food Spoilage and Natural-Based Strategies for its Inhibition. Food Res. Int. 127, 108754. doi:10.1016/j.foodres.2019.108754

McMichael, A. J. (2004). Environmental and Social Influences on Emerging Infectious Diseases: Past, Present and Future. Philos. Trans. R. Soc. Lond. B Biol. Sci. 359 (1447), 1049-1058. doi:10.1098/rstb.2004.1480
Mo, W. Y., Man, Y. B., and Wong, M. H. (2018). Use of Food Waste, Fish Waste and Food Processing Waste for China's Aquaculture Industry: Needs and challenge. Sci. Total Environ. 613-614, 635-643. doi:10.1016/ j.scitotenv.2017.08.321

Newman, D. J., and Cragg, G. M. (2012). Natural Products as Sources of New Drugs over the 30 Years from 1981 to 2010. J. Nat. Prod. 75 (3), 311-335. doi:10.1021/ np200906s

Oh, D. R., Kim, J. R., and Kim, Y. R. (2010). Genistein Inhibits Vibrio Vulnificus Adhesion and Cytotoxicity to HeLa Cells. Arch. Pharm. Res. 33 (5), 787-792. doi:10.1007/s12272-010-0520-y

Packiavathy, I. A., Sasikumar, P., Pandian, S. K., and Veera Ravi, A. (2013). Prevention of Quorum-sensing-mediated Biofilm Development and Virulence Factors Production in Vibrio Spp. By Curcumin. Appl. Microbiol. Biotechnol. 97 (23), 10177-10187. doi:10.1007/s00253-013-4704-5

Palumbi, S. R. (2001). Humans as the World's Greatest Evolutionary Force. Science 293 (5536), 1786-1790. doi:10.1126/science.293.5536.1786

Patel, B., Kumari, S., Banerjee, R., Samanta, M., and Das, S. (2017). Disruption of the Quorum Sensing Regulated Pathogenic Traits of the Biofilm-Forming Fish Pathogen Aeromonas Hydrophila by Tannic Acid, a Potent Quorum Quencher. Biofouling 33 (7), 580-590. doi:10.1080/08927014.2017.1336619

Pérez-Sánchez, T., Mora-Sánchez, B., and Balcázar, J. L. (2018). Biological Approaches for Disease Control in Aquaculture: Advantages, Limitations and Challenges. Trends Microbiol. 26 (11), 896-903. doi:10.1016/j.tim.2018.05.002

Rama Devi, K., Srinivasan, R., Kannappan, A., Santhakumari, S., Bhuvaneswari, M., Rajasekar, P., et al. (2016). In Vitro and In Vivo Efficacy of Rosmarinic Acid on Quorum Sensing Mediated Biofilm Formation and Virulence Factor Production in Aeromonas Hydrophila. Biofouling 32 (10), 1171-1183. doi:10.1080/08927014.2016.1237220

Rasko, D. A., and Sperandio, V. (2010). Anti-virulence Strategies to Combat Bacteria-Mediated Disease. Nat. Rev. Drug Discov. 9 (2), 117-128. doi:10.1038/ $\operatorname{nrd} 3013$

Ruiz-Larrea, M. B., Mohan, A. R., Paganga, G., Miller, N. J., Bolwell, G. P., and RiceEvans, C. A. (1997). Antioxidant Activity of Phytoestrogenic Isoflavones. Free Radic. Res. 26 (1), 63-70. doi:10.3109/10715769709097785

Savoia, D. (2012). Plant-derived Antimicrobial Compounds: Alternatives to Antibiotics. Future Microbiol. 7 (8), 979-990. doi:10.2217/fmb.12.68

Singh, V., Rathore, G., Kapoor, D., Mishra, B. N., and Lakra, W. S. (2008). Detection of Aerolysin Gene in Aeromonas hydrophila Isolated from Fish and Pond Water. Indian J Microbiol. 48 (4), 453-458. doi:10.1007/s12088-008-0056-8

Siriviriyakul, P., Werawatganon, D., Phetnoo, N., Somanawat, K., Chatsuwan, T., Klaikeaw, N., et al. (2020). Genistein Attenuated Gastric Inflammation and Apoptosis in Helicobacter Pylori-Induced Gastropathy in Rats. BMC Gastroenterol. 20 (1), 410. doi:10.1186/s12876-020-01555-x

Spagnuolo, C., Russo, G. L., Orhan, I. E., Habtemariam, S., Daglia, M., Sureda, A., et al. (2015). Genistein and Cancer: Current Status, Challenges, and Future Directions. Adv. Nutr. 6 (4), 408-419. doi:10.3945/an.114.008052

Swift, S., Lynch, M. J., Fish, L., Kirke, D. F., Tomás, J. M., Stewart, G. S., et al. (1999). Quorum sensing-dependent Regulation and Blockade of Exoprotease Production in Aeromonas Hydrophila. Infect. Immun. 67 (10), 5192-5199. doi:10.1128/IAI.67.10.5192-5199.1999

Tan, E., Low, K. W., Wong, W. S., and Leung, K. Y. (1998). Internalization of Aeromonas Hydrophila by Fish Epithelial Cells Can Be Inhibited with a Tyrosine Kinase Inhibitor. Microbiology (Reading) 144 (Pt 2), 299-307. doi:10.1099/00221287-144-2-299

Tanhay Mangoudehi, H., Zamani, H., Shahangian, S. S., and Mirzanejad, L. (2020). Effect of Curcumin on the Expression of ahyI/R Quorum Sensing Genes and Some Associated Phenotypes in Pathogenic Aeromonas Hydrophila Fish Isolates. World J. Microbiol. Biotechnol. 36 (5), 70. doi:10.1007/s11274-02002846-x

Valladão, G. M., Gallani, S. U., and Pilarski, F. (2015). Phytotherapy as an Alternative for Treating Fish Disease. J. Vet. Pharmacol. Ther. 38 (5), 417-428. doi:10.1111/jvp.12202

Vela, E. M., Bowick, G. C., Herzog, N. K., and Aronson, J. F. (2008). Genistein Treatment of Cells Inhibits Arenavirus Infection. Antivir. Res. 77 (2), 153-156. doi:10.1016/j.antiviral.2007.09.005

Velázquez, L. D., Escudero, M. E., and de Guzmán, A. M. (2001). Antibacterial Effects of Different Food-Related Phosphates Using Aeromonas Hydrophila. J. Food Prot. 64 (2), 195-200. doi:10.4315/0362-028x-64.2.195 
Verdrengh, M., Collins, L. V., Bergin, P., and Tarkowski, A. (2004). Phytoestrogen Genistein as an Anti-staphylococcal Agent. Microbes Infect. 6 (1), 86-92. doi:10.1016/j.micinf.2003.10.005

Wei, H., Wei, L., Frenkel, K., Bowen, R., and Barnes, S. (1993). Inhibition of Tumor Promoter-Induced Hydrogen Peroxide Formation In Vitro and In Vivo by Genistein. Nutr. Cancer 20 (1), 1-12. doi:10.1080/01635589309514265

Wuethrich, I., Peeters, J. G., Blom, A. E., Theile, C. S., Li, Z., Spooner, E., et al. (2014). SiteSpecific Chemoenzymatic Labeling of Aerolysin Enables the Identification of New Aerolysin Receptors. Plos One 9 (10), e109883. doi:10.1371/journal.pone.0109883

Yousefi Jourdehi, A., Sudagar, M., Bahmani, M., Hosseini, S. A., Dehghani, A. A., and Yazdani, M. A. (2014). Comparative Study of Dietary Soy Phytoestrogens Genistein and Equol Effects on Growth Parameters and Ovarian Development in Farmed Female Beluga sturgeon, Huso huso. Fish. Physiol. Biochem. 40 (1), 117-128. doi:10.1007/s10695-013-9829-z

Zhao, X. L., Wu, G., Chen, H., Li, L., and Kong, X. H. (2020). Analysis of Virulence and Immunogenic Factors in Aeromonas Hydrophila: Towards the Development of Live Vaccines. J. Fish. Dis. 43 (7), 747-755. doi:10.1111/jfd.13174
Conflict of Interest: The authors declare that the research was conducted in the absence of any commercial or financial relationships that could be construed as a potential conflict of interest.

Publisher's Note: All claims expressed in this article are solely those of the authors and do not necessarily represent those of their affiliated organizations, or those of the publisher, the editors and the reviewers. Any product that may be evaluated in this article, or claim that may be made by its manufacturer, is not guaranteed or endorsed by the publisher.

Copyright (C) 2021 Dong, Zhang, Li, Liu, Zhou, Yang, Xu, Yang and Ai. This is an open-access article distributed under the terms of the Creative Commons Attribution License (CC BY). The use, distribution or reproduction in other forums is permitted, provided the original author(s) and the copyright owner(s) are credited and that the original publication in this journal is cited, in accordance with accepted academic practice. No use, distribution or reproduction is permitted which does not comply with these terms. 